\title{
Impact de la solifluxion sur les niveaux archéologiques : simulation à partir d'une expérience en milieu actif et application à des sites paléolithiques aquitains
}

Archaeological record transformation by solifluction: computer simulation and application to prehistoric sites from South-West France

\section{A. Lenoble, P. Bertran, F. Lacrampe, L. Bourguignon et L. Detrain}

\section{OpenEdition}

\section{Journals}

Édition électronique

URL : http://journals.openedition.org/paleo/1255

DOI : $10.4000 /$ paleo. 1255

ISSN : 2101-0420

Éditeur

SAMRA

Édition imprimée

Date de publication : 1 décembre 2003

Pagination : 105-122

ISSN : 1145-3370

Référence électronique

A. Lenoble, P. Bertran, F. Lacrampe, L. Bourguignon et L. Detrain, « Impact de la solifluxion sur les niveaux archéologiques : simulation à partir d'une expérience en milieu actif et application à des sites paléolithiques aquitains », PALEO [En ligne], 15 | 2003, mis en ligne le 02 août 2010, consulté le 07 juillet 2020. URL : http://journals.openedition.org/paleo/1255; DOI : https://doi.org/10.4000/paleo. 1255

Ce document a été généré automatiquement le 7 juillet 2020.

PALEO est mis à disposition selon les termes de la licence Creative Commons Attribution - Pas d'Utilisation Commerciale - Pas de Modification 4.0 International. 


\title{
Impact de la solifluxion sur les
} niveaux archéologiques : simulation à partir d'une expérience en milieu actif et application à des sites paléolithiques aquitains

\author{
Archaeological record transformation by solifluction: computer simulation and \\ application to prehistoric sites from South-West France
}

A. Lenoble, P. Bertran, F. Lacrampe, L. Bourguignon et L. Detrain

\section{Introduction}

Depuis les remarques d'Ascher (1968) et les travaux de Schiffer (1972), l'idée que tous les sites archéologiques ont subi à des degrés divers des transformations après leur abandon par l'Homme s'est très largement imposée (Schiffer 1983, 1987 ; Binford 1981 ; Butzer 1982 ; Bertran 1994 ; Waters 1992). De manière à apprécier ces transformations, plusieurs types d'approches taphonomiques ont été développées. L'état de surface et la fragmentation des différents types de vestiges, leur composition granulométrique, la fabrique (orientation et inclinaison des objets), la distribution des remontages et la répartition spatiale du matériel archéologique font partie des critères les plus communément utilisés dans ce but. L'interprétation des résultats obtenus est fondée sur une comparaison avec des référentiels actuels, naturels ou issus de l'expérimentation. Certains aspects, comme les transformations provoquées par les processus sédimentaires et biologiques sur la répartition des vestiges, n'ont cependant fait l'objet que d'un nombre limité de travaux. Ils concernent essentiellement le domaine fluviatile, documenté par les expériences de Schick (1986), les perturbations provoquées par les labours (Steinberg 1996), la reptation liée à la formation d'aiguilles de glace en surface (Bowers et al., 1983) et l'activité de la faune du sol (Cahen et 
Moeyersons 1977 ; Johnson 2002 ; Araujo et Marcelino 2003). L'une des principales raisons est la lourdeur des expériences à mettre en œuvre pour rendre compte des configurations archéologiques. Une des limitations de la démarche expérimentale réside en effet dans la durée des expériences, qui est souvent trop courte pour aboutir à des résultats directement applicables à l'archéologie. Le caractère opératoire des modèles expérimentaux varie cependant beaucoup en fonction des mécanismes sédimentaires étudiés. Comme le montrent les travaux de Schick (1986), les transformations subies par des ensembles archéologiques en milieu fluviatile sont principalement liées à quelques événements efficaces et peuvent être ainsi appréhendées de manière relativement fiable au cours d'expériences limitées dans le temps. Dans ce type de milieu, seul un enfouissement rapide prévient la destruction complète d'un site. A l'inverse, d'autres mécanismes comme la reptation sont caractérisés par la répétition pendant une longue période de déplacements minimes, de l'ordre de quelques millimètres ou centimètres par an, qui affectent collectivement l'ensemble des objets archéologiques. L'expérimentation seule ne permet donc pas de décrire de manière adéquate la nature des déformations provoquées par ces mécanismes. De ce fait, la mise en évidence de tels phénomènes sur un site est généralement traitée de manière intuitive.

Dans les sites paléolithiques du sud-ouest de la France, les témoignages de phénomènes périglaciaires et en particulier de la solifluxion, c'est-à-dire de la reptation du sol due aux alternances de gel et de dégel, ont fréquemment été observés (Bertran, 1994 ; Texier, 2001 ; Couchoud, 2002). Cependant, en l'absence de modèle adapté, l'impact de ce processus sur la formation des niveaux archéologiques reste mal compris. Ce constat s'est posé avec acuité lors de l'étude de deux sites du Paléolithique moyen, Petit-Bost et Croix-de-Canard, récemment découverts à l'occasion des travaux d'archéologie préventive sur le tracé de l'autoroute A89 à proximité de Périgueux (Dordogne, France). Pour ces deux sites, l'étude géoarchéologique a montré que la solifluxion avait probablement joué un rôle significatif dans la mise en place des dépôts.

3 La démarche expérimentale ne permettant pas une approche correcte de ce processus, le recours à une simulation informatique basée sur des données expérimentales s'est imposée pour obtenir un modèle qui puisse rendre compte des situations archéologiques rencontrées à Petit-Bost et Croix-de-Canard. Cette démarche, comparable à celle mise en œuvre par Bowers et al., (1983), s'est appuyée sur les résultats du programme TRANSIT (Transfert de Référentiels Actuels de l'étage Nival aux SITes paléolithiques) qui s'est déroulé en milieu périglaciaire alpin (Texier et al., 1998 ; Todisco et al., 2000).

Ce travail se propose donc d'exposer les résultats d'une simulation des déformations occasionnées par la solifluxion, d'en dégager des implications sur la répartition des vestiges, puis de confronter les configurations obtenues à celles observées sur les sites de Petit-Bost et de Croix-de-Canard. Ces deux sites sont d'abord présentés.

\section{Données archéologiques : les sites de Croix-de-Canard et de Petit-Bost}


Figure 1 - Localisation des sites.

Figure 1 - Sites location.

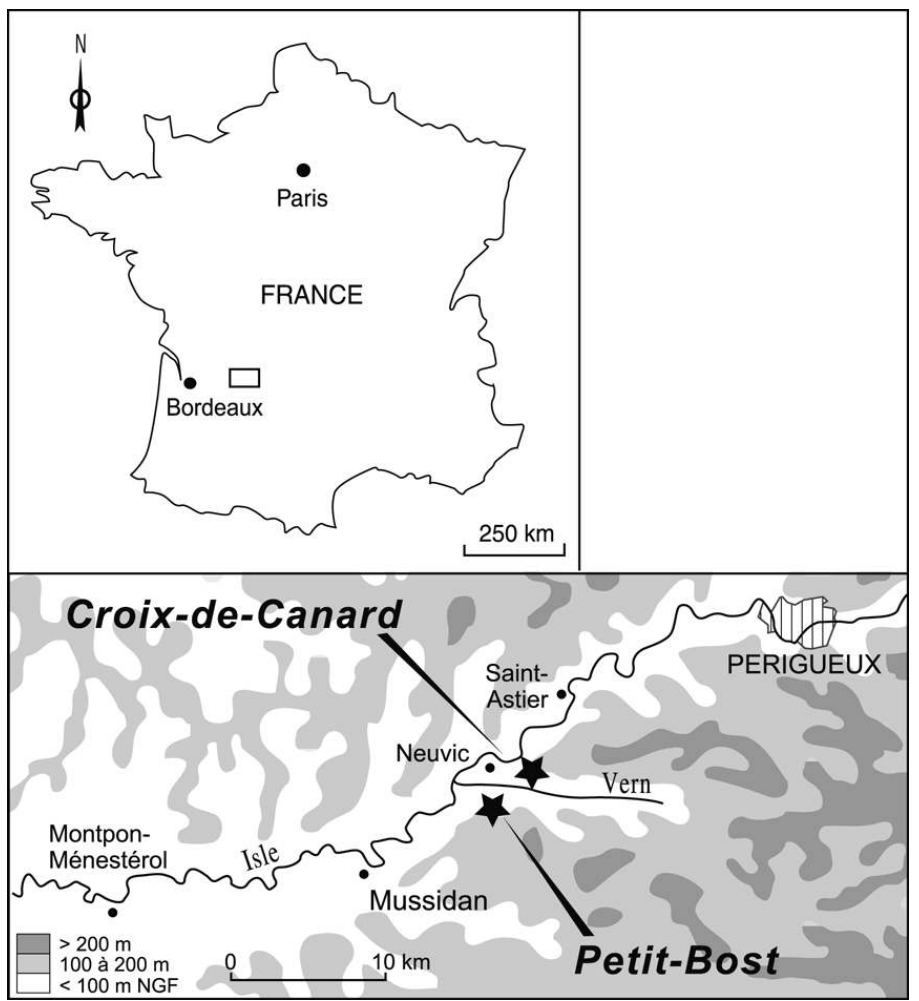

5 Le site de Croix-de-Canard (fouilles L. Detrain) est localisé sur une terrasse de la vallée de l'Isle à une trentaine de kilomètres à l'ouest de Périgueux (fig. 1). Plusieurs niveaux du Paléolithique moyen y ont été mis en évidence. Le plus récent (" secteur 2 "), qui est le seul abordé dans cette étude, a été trouvé à environ 0,60 $\mathrm{m}$ de profondeur dans des colluvions sablo-argileuses. La pente de la nappe de vestiges atteint $3^{\circ}$. Le faible enfouissement du site laissant suspecter un rôle important des processus naturels dans la constitution du niveau archéologique, une étude taphonomique détaillée a été entreprise. Les principales conclusions de cette étude sont les suivantes (Detrain et al. rapport en cours ; Bertran et Lenoble 2002) :

- Les objets présentent une orientation préférentielle très marquée selon la pente (fig. 2). Une telle orientation préférentielle n'a jamais été observée dans des sites archéologiques non perturbés ; elle entre en revanche dans le champ des valeurs connues pour la solifluxion (fig. 3). L'hypothèse de transformations importantes par la dynamique de versant au cours des phases froides du Pléistocène récent apparaît donc très vraisemblable.

- L'absence de véritable concentration et la faible densité des vestiges plaident également en faveur d'une dispersion sur une grande surface d'unités archéologiques initialement mieux délimitées. Malgré cela, un fort taux de remontage a été trouvé. Il atteint $37 \%$ du total du matériel récolté, valeur comparable à celle connue dans de nombreux sites d'habitats paléolithiques. 
Figure 2 - Fabrique des éclats allongés, Croix-de-Canard, secteur 2. Stéréogramme de Schmidt, hémisphère inférieure.

Figure 2 - Fabric of elongated flakes, Croix-de-Canard, sector 2. Schmidt stereogram, lower hemisphere.

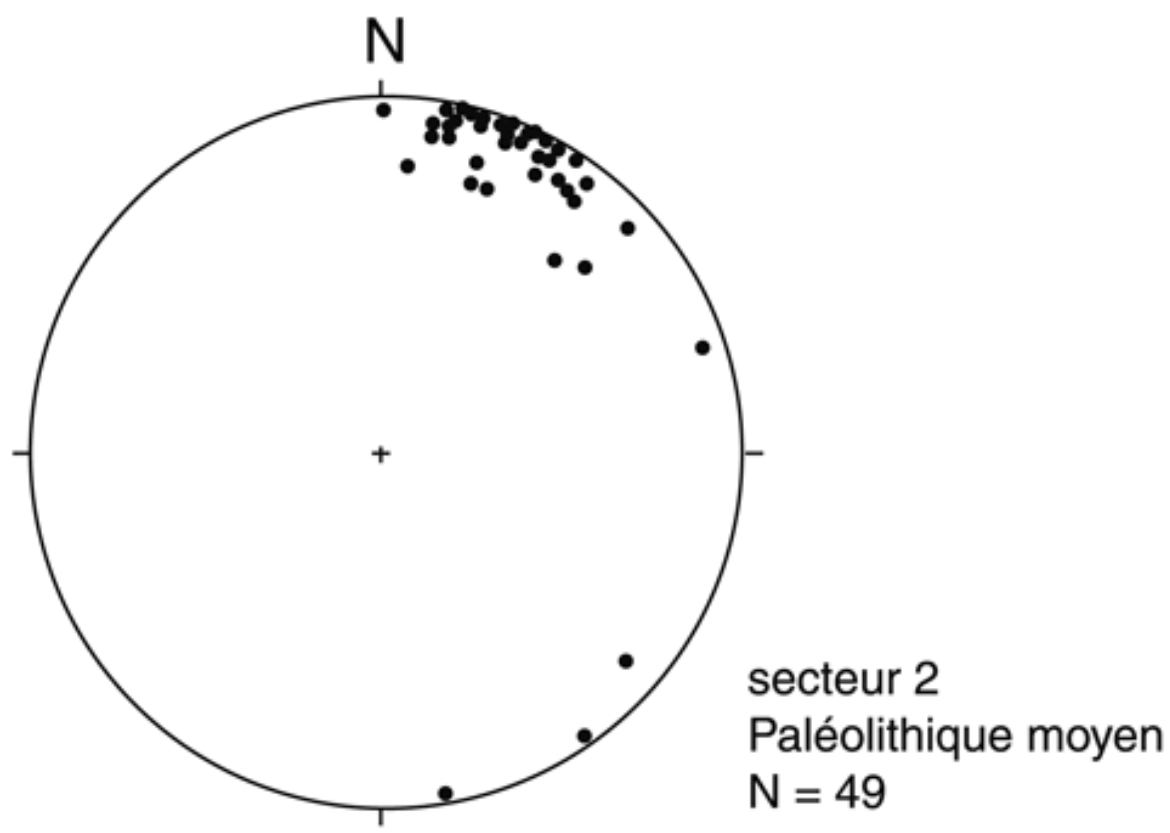


Figure 3 - Fabrique des niveaux archéologiques de Croix-de-Canard, secteur 2 (1 et 2) et de PetitBost (3). Sur ce diagramme, les aires caractéristiques des différents processus de perturbation sont indiquées d'après Bertran et Lenoble (2002). Les valeurs propres normalisées de la fabrique, $E 1$, E2 et E3, sont utilisées pour établir les indices d'élongation (EL) et d'isotropie (IS) de Benn (1994).

Figure 3 - Fabric of Croix-de-Canard, sector 2 (1 and 2), and Petit-Bost (3). The areas typifying the processes of disturbance are indicated according to Bertran and Lenoble (2002). The Fabric Eigenvalue vectors, E1, E2 and E3, are used for calculation of elongation (EL) and isotropy index (IS) according to Benn (1994).

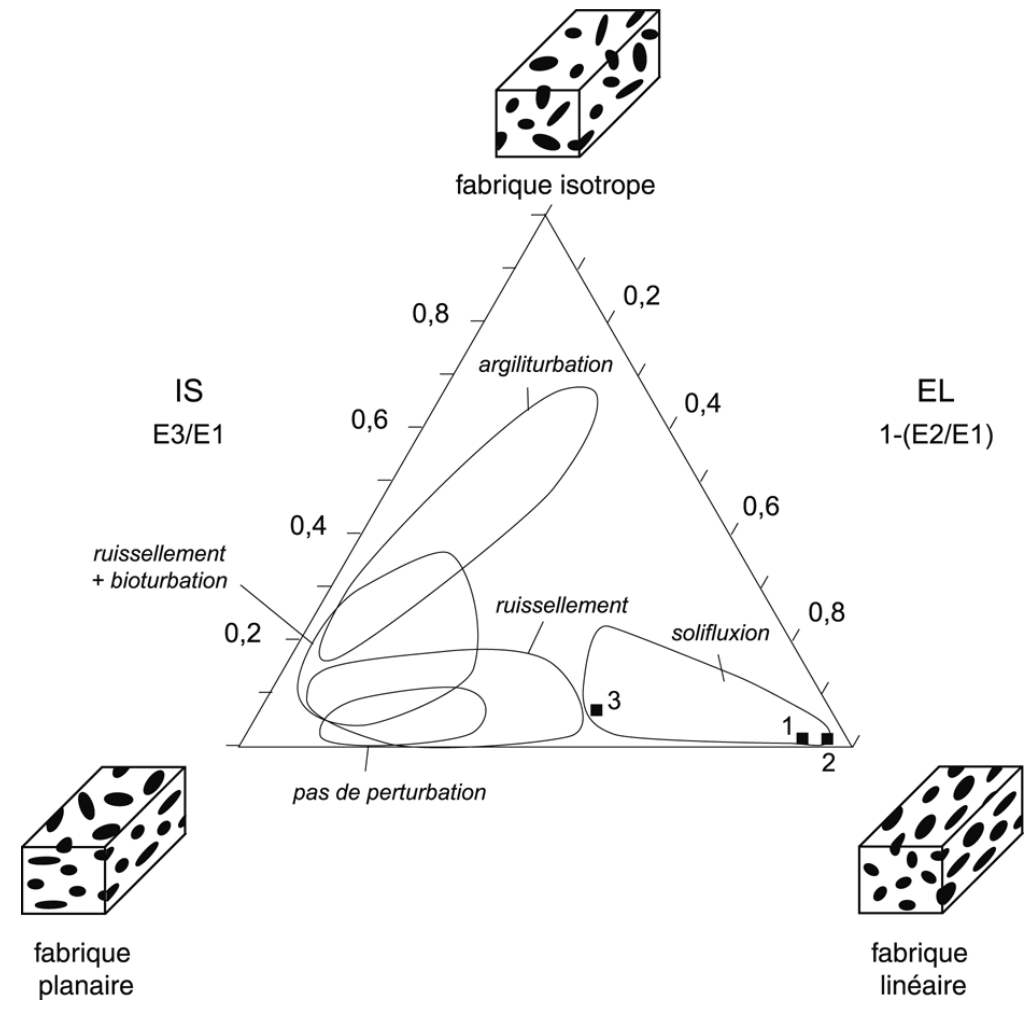

6 L'hypothèse d'une altération importante de la physionomie initiale du site par la solifluxion a donc été retenue à l'issue de l'étude taphonomique, bien que l'intégrité du matériel lithique semble avoir été en grande partie préservée.

7 Le site de Petit-Bost (fouilles L. Bourguignon) est localisé dans un contexte identique à environ $3 \mathrm{~km}$ à l'Ouest du précédent. Le principal niveau archéologique («niveau 1») présente une pente de $4^{\circ}$. Il a été trouvé à environ $1,3 \mathrm{~m}$ de profondeur dans des colluvions sablo-argileuses recouvrant la terrasse alluviale. L'analyse taphonomique met en évidence les points suivants (Bourguignon et al., rapport en cours) :

- Comme à Croix-de-Canard, les objets présentent une orientation préférentielle très significative, qui suggère un déplacement par solifluxion sur le versant (fig. 3). L'abondance des traces liées à la présence de glace dans le sol (pseudomorphoses de coins de glace, structures lamellaires dues à de la glace de ségrégation en lentilles) appuie cette hypothèse. - De façon contradictoire et à l'opposé de ce qui a été observé à Croix-de-Canard, les données archéologiques suggèrent que les transformations subies par le site ont été négligeables. Les arguments consistent en :

1) une forte densité locale du matériel lithique et le caractère relativement bien délimité des concentrations, ce qui suppose que la répartition des vestiges n'ait pas été affectée par un phénomène d'homogénéisation par les processus naturels (fig. 4);

2) la présence de plusieurs remontages représentant $20 \%$ de l'ensemble de pièces et pouvant mettre en jeu un nombre important de pièces (118 pour le plus important). Ces remontages 
sont essentiellement regroupées au sein d'une unique concentration. Celle-ci est interprétée comme une aire de débitage, sans que l'on puisse à proprement parler d'amas en raison de sa forme allongée et de ses grandes dimensions.

Figure 4 - Plan de répartition des vestiges du niveau 1 de Petit-Bost. Les pointillés délimitent le contour des tranchées de reconnaissance.

Figure 4 - Artefact distribution map of level 1, Petit-Bost. The dotted lines indicate the location of the survey trenches.

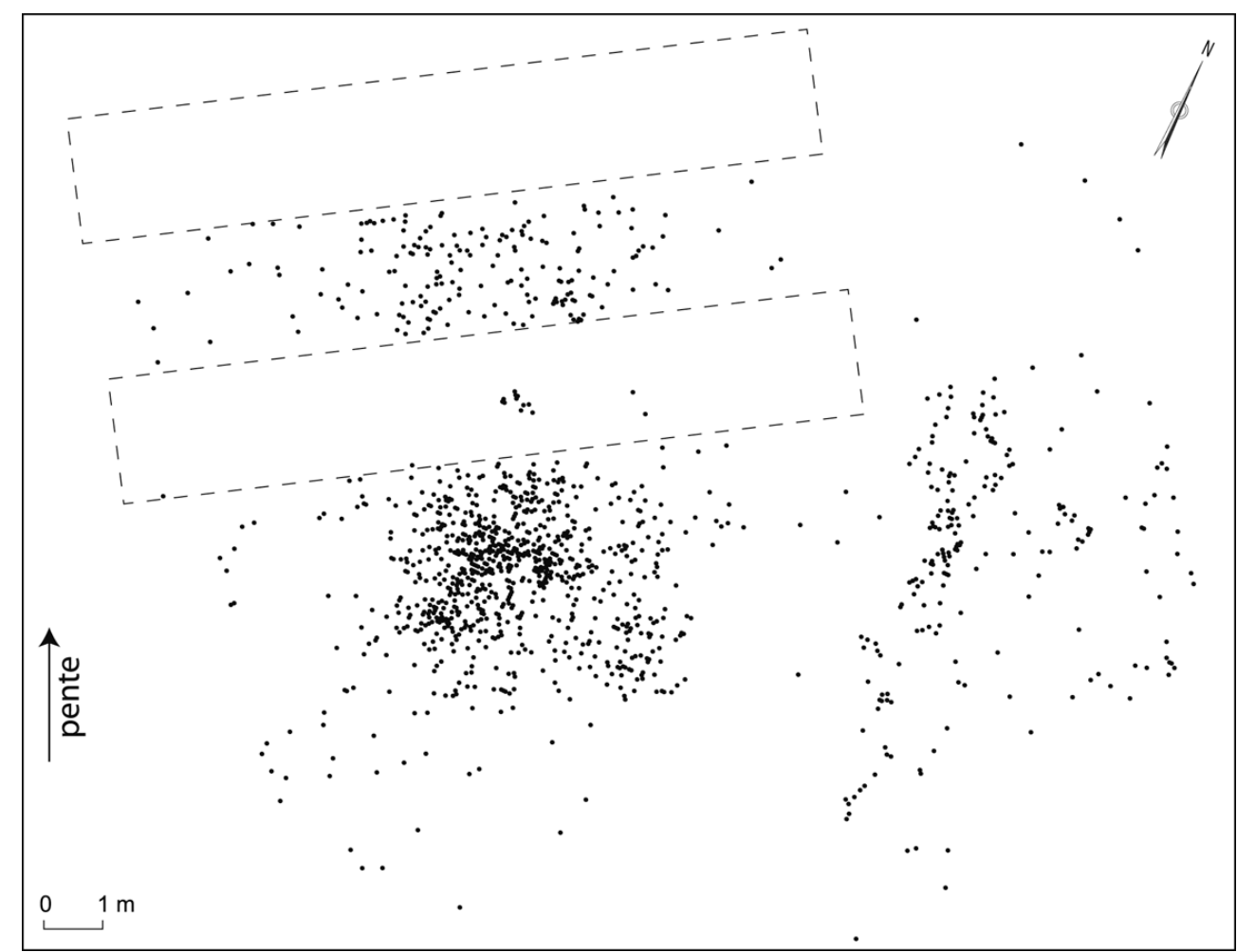

Pour le site de Petit-Bost, l'étude taphonomique bute donc à ce stade de l'analyse sur le constat d'une coexistence dans un même niveau archéologique de caractères à la fois d'origine indéniablement anthropique, en particulier en ce qui concerne la répartition spatiale des vestiges, et d'indices de perturbations par des processus naturels, en l'occurrence par la solifluxion.

\section{L'expérience transit}

L'expérience TRANSIT a été conçue pour documenter les transformations occasionnées par les processus périglaciaires sur des assemblages lithiques et osseux. Pour cela, différentes expériences ont été implantées sur un versant du massif de la Mortice, dans les Alpes méridionales françaises, à $3075 \mathrm{~m}$ d'altitude (fig. 5). Le climat est caractérisé par une température moyenne annuelle d'environ $-3^{\circ} \mathrm{C}$, ce qui suppose la présence d'un pergélisol et des précipitations comprises entre 1500 et $2000 \mathrm{~mm}$. La dynamique de versant, typique de l'étage périglaciaire, est dominée par la solifluxion et se traduit par la formation de coulées à front pierreux d'épaisseur semi-métrique qui progressent vers l'aval à raison de quelques centimètres par an (fig. 6). Localement et de manière temporaire, d'autres processus sédimentaires peuvent être actifs. Ils correspondent à des transports de sables et de plaquettes de schiste par le vent, ainsi qu'à des incisions 
par le ruissellement au cours des orages estivaux. Ces processus ne laissent que peu de traces dans l'enregistrement sédimentaire, dans la mesure où les dépôts associés sont rapidement repris par la solifluxion et intégrés aux coulées. En coupe, les dépôts présentent une stratification plus ou moins bien exprimée, qui reflète l'empilement progressif des coulées successives (Bertran et al. 1993, 1995).

Figure 5 - Vue générale du versant est de la Mortice. On distingue des traînées parallèles à la pente qui correspondent aux coulées de solifluxion.

Figure 5 - General view of the east-facing slope of la Mortice. Slope-parallel tracks correspond to solifluction lobes.

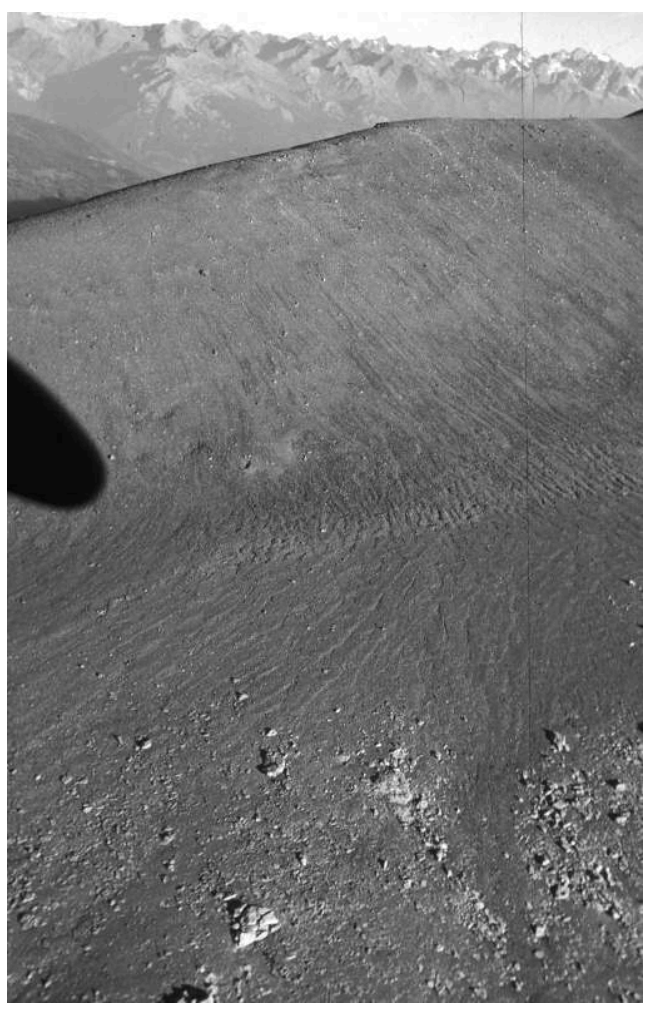


Figure 6 - Vue d'une coulée de solifluction à front pierreux, site de La Mortice, Alpes Françaises. Cette coulée, composée de flysch de couleur brune, progresse lentement sur des matériaux calcaires gris.

Figure 6 - Stone-banked solifluction lobe at La Mortice. This lobe is mainly composed of brown schist fragments and moves slowly over grey limestone debris.

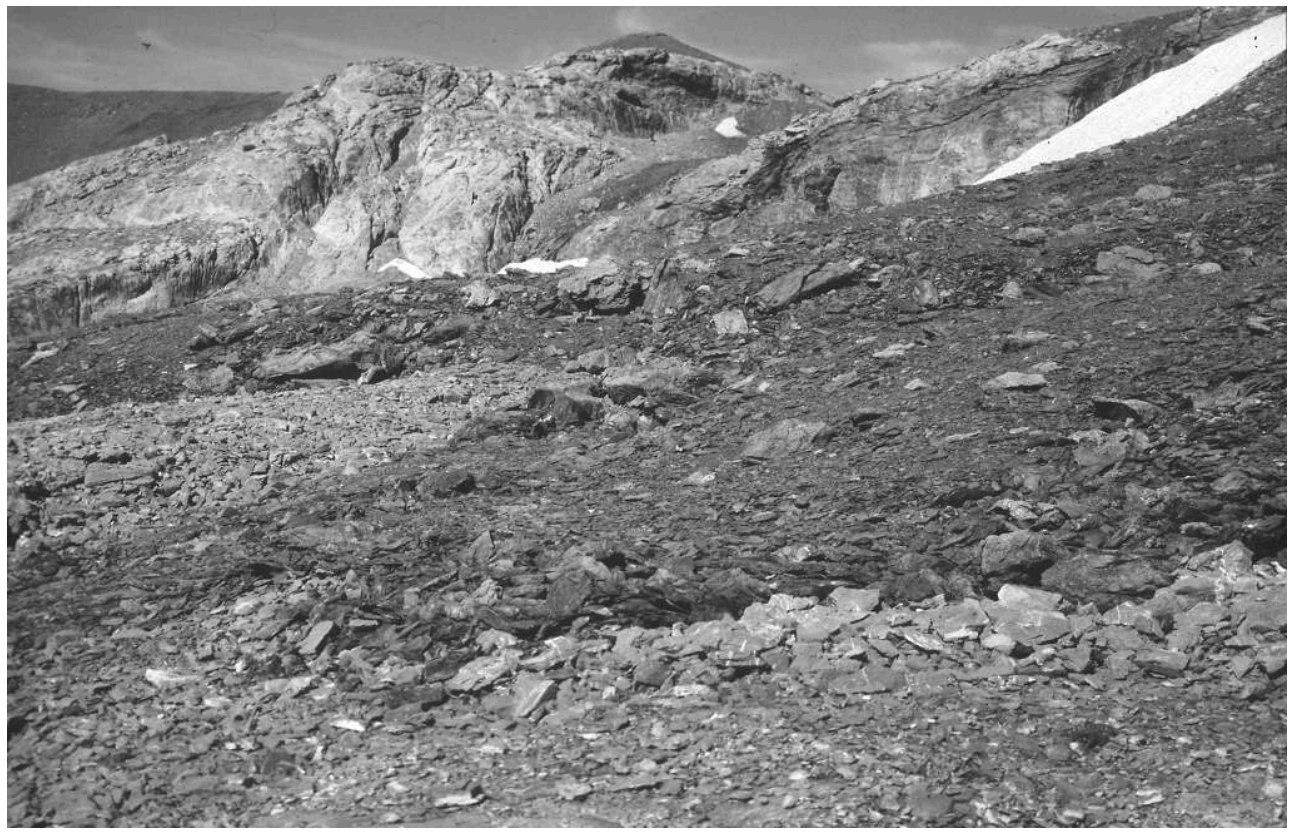

Parmi les expériences effectuées (Texier et al. 1998), l'une d'entre elles a été consacrée à l'enregistrement des déplacements à la surface des coulées, sur des pentes de 8 à $14^{\circ}$. Les objets marqués étaient répartis dans cinq cellules différentes, dont les mouvements ont été suivis sur une période de cinq ans (1991-1996) (fig. 7 et 8). Ces déplacements sont principalement provoqués par les alternances de gonflement et d'affaissement du sol liés à la cristallisation de glace en lentilles (cryoreptation), par le soulèvement et la reptation des graviers dus à la formation d'aiguilles de glace en surface (pipkrakes), ainsi que par les déformations liées à la sursaturation en eau au dégel (gélifluxion) (Coutard et Ozouf 1996). 
Figure 7 - Vue d'une cellule expérimentale de l'expérience Transit en cours d'implantation. Figure 7 - Close-up view of an experimental cell during emplacement.

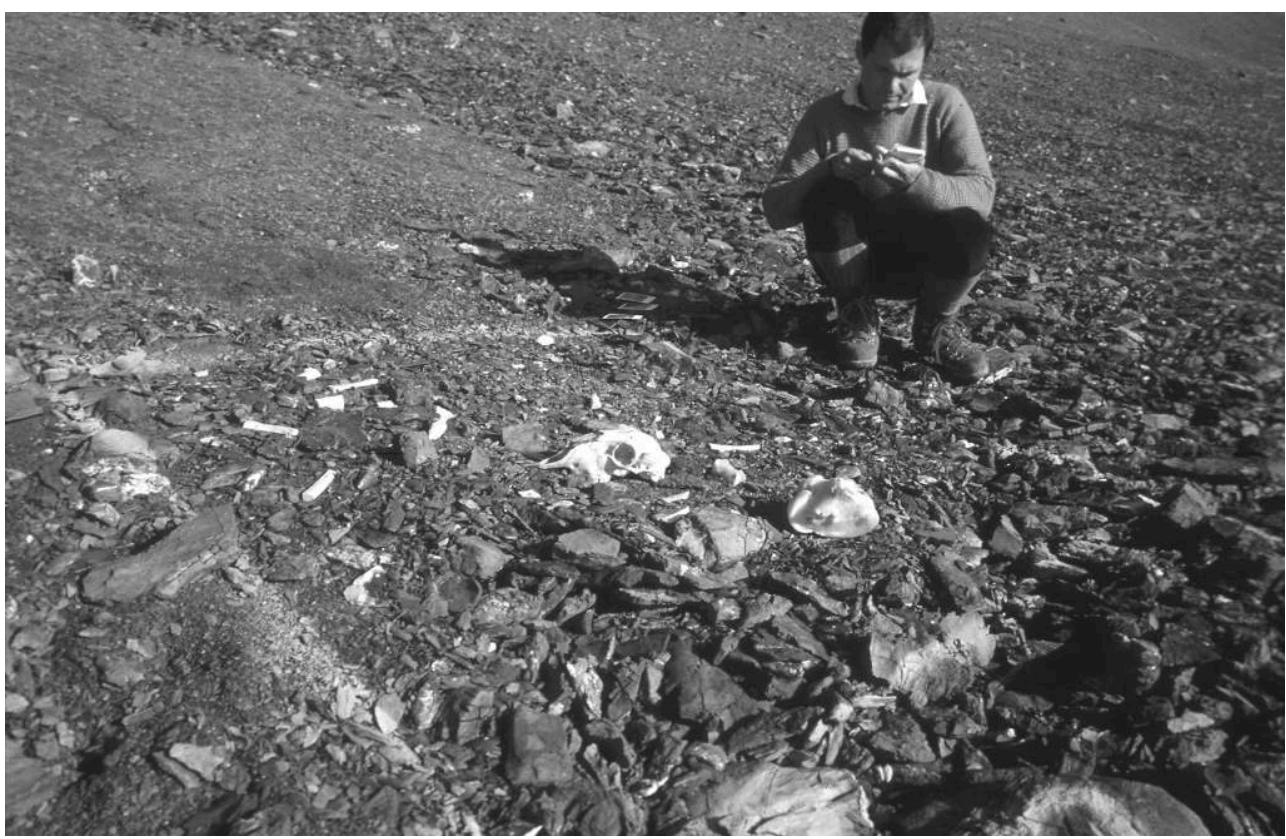

Figure 8 - Vue d'une cellule de l'expérience Transit après 3 années d'expérience. Une partie des objets initialement localisés à l'intérieur du carré jaune ont migré vers l'extérieur. Figure 8 - View of a cell after 3 years of experiment. Some artefacts that were initially located inside the yellow square are now outside.

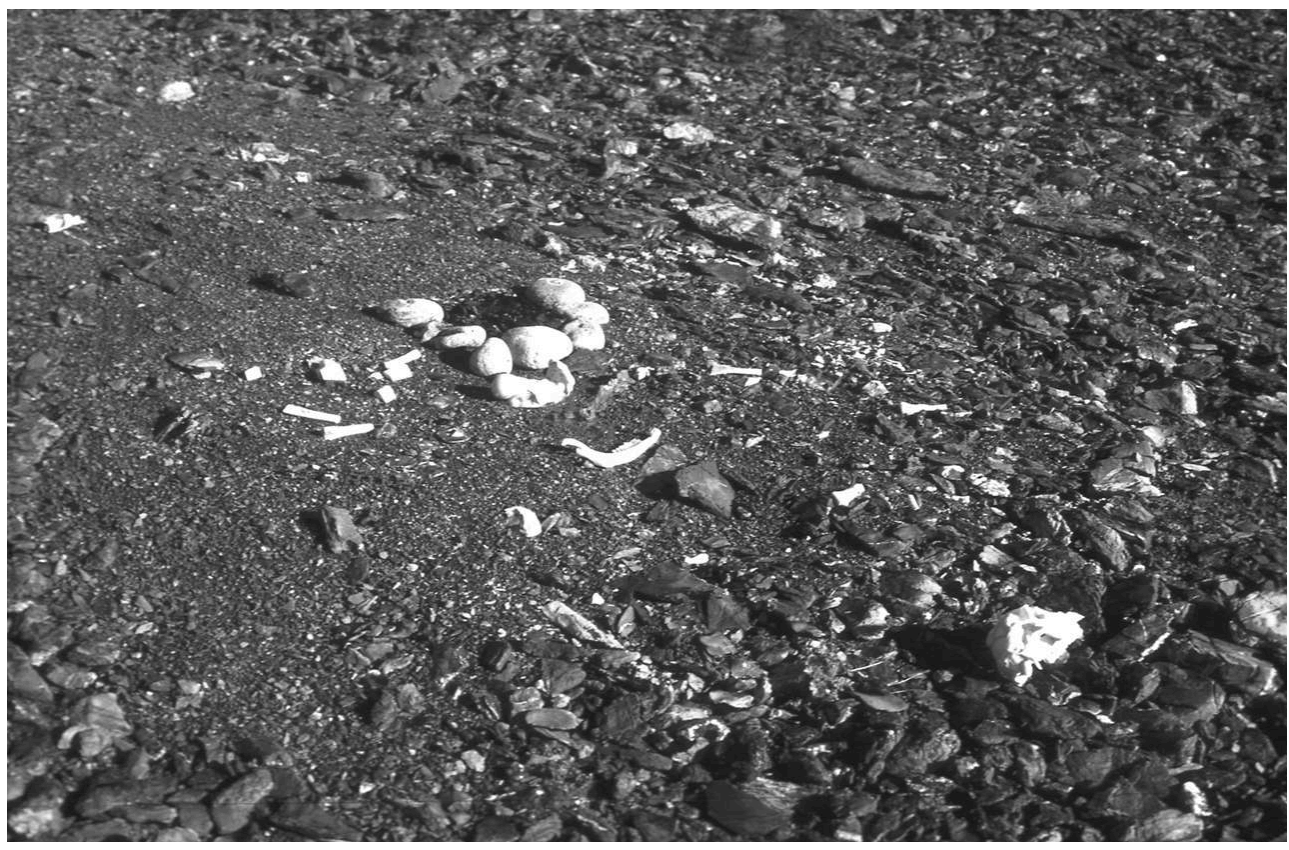

11 Une caractérisation statistique des déplacements a été menée par Todisco (1999) et Todisco et al. (2000). Ce travail montre que les déplacements annuels moyens sont de l'ordre de quelques centimètres pour toutes les cellules (moyennes sur 5 ans comprises entre 1,8 et 3,6 cm/an) (fig. 9). Parmi les mouvements enregistrés, rares sont ceux qui dépassent une quinzaine de centimètres. Une variabilité interannuelle significative, essentiellement corrélée avec le nombre de jours de pluie au cours des mois d'été, 
apparaît également. En raison de la haute altitude, les alternances de gel-dégel se produisent en effet surtout durant les mois de juillet, août et septembre, le sol restant gelé ou couvert de neige le reste de l'année. Pendant la période estivale, l'efficacité des gels nocturnes (c'est-à-dire leur capacité à donner naissance à de la glace de ségrégation dans le sol) est contrôlée par l'humidité du sol, elle-même tributaire de la pluie tombée les jours précédents.

Figure 9 - A - Déplacement des silex de la cellule 1 enregistrés de 1991 à 1995 à la Mortice au cours de l'expérience TRANSIT. La longueur des flèches est proportionnelle au mouvement. B Distribution des déplacements.

Figure 9 - A - Artefact displacements recorded from 1991 to 1995 at cell 1, TRANSIT experiment, la Mortice. The length of the arrows is proportional to the movement. B - Distribution of the displacements.

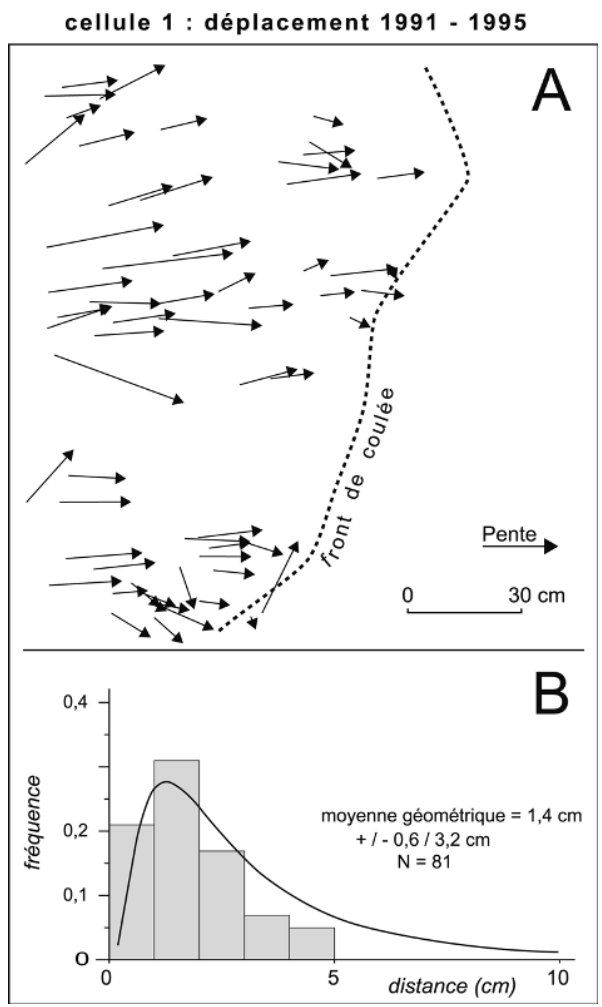

Quelques déplacements dépassent cependant d'un ou deux ordres de grandeur les valeurs moyennes et peuvent atteindre $1 \mathrm{~m}$ en une année (fig. 10). Ils sont considérés comme «accidentels», en ce sens que la distance parcourue est trop importante pour être expliquée par la seule solifluxion. Ces déplacements, qui concernent préférentiellement les charbons de bois et les ossements, sont attribués à l'action du ruissellement, de la grêle et/ou du vent. Une variabilité spatiale significative a également été constatée. Typiquement, les éléments marqués disposés sur les fronts et les bords pierreux des coulées progressent plus lentement que ceux qui reposent sur la partie centrale à texture plus fine. 
Figure 10 - Distribution des déplacements "accidentels" dans l'expérience TRANSIT, toutes cellules confondues.

Figure 10 - 'Accidental' displacements distribution, TRANSIT experiment, all cells mixed.
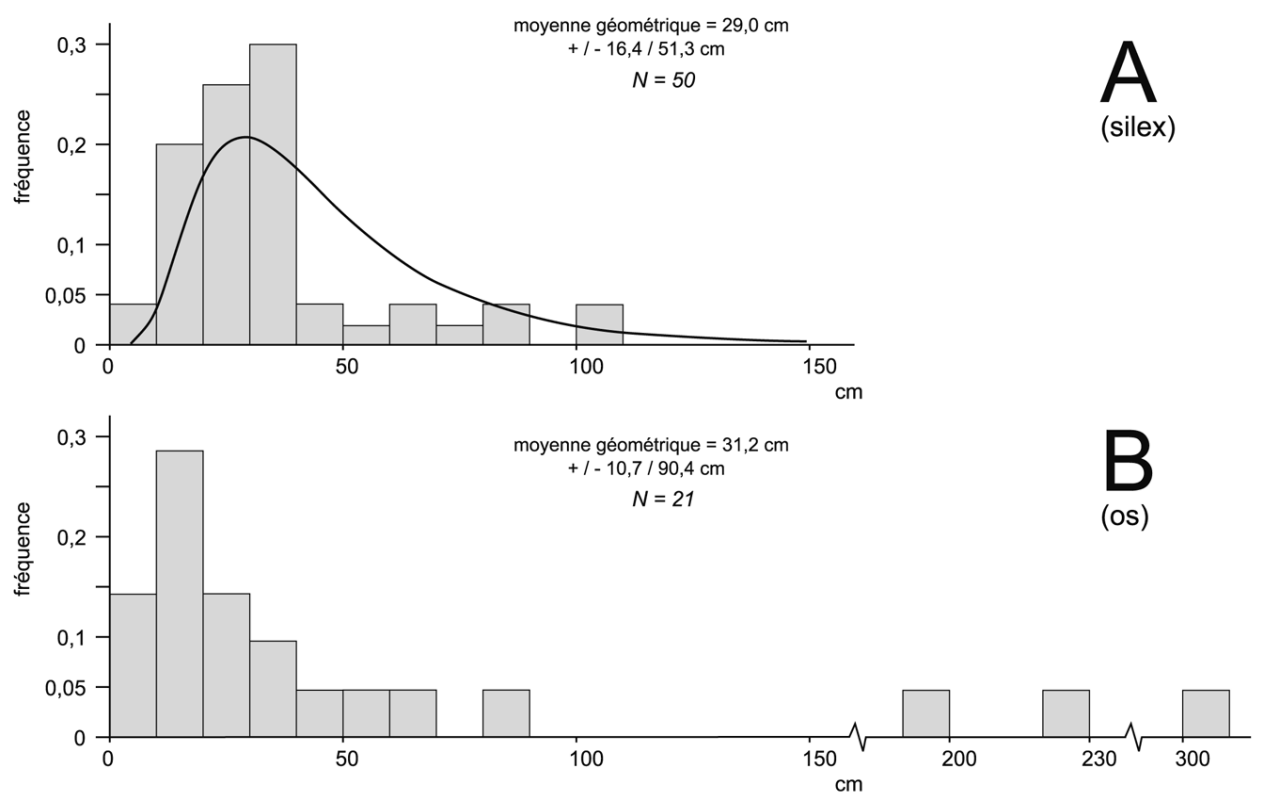

\section{Principes de la simulation}

13 Le principe de la simulation consiste à programmer des déplacements dont les caractéristiques statistiques (longueur, orientation) sont identiques à celles des déplacements mesurés dans l'expérimentation. Cette démarche prend donc en compte la variabilité importante des mesures, qui joue un grand rôle dans les processus de dispersion des objets.

La distribution des mouvements liés à la solifluxion suit une loi log-normale. Elle peut alors être caractérisée par la moyenne géométrique et l'écart-type des valeurs transformées en log base 10 (Caine 1968). Les déplacements accidentels ont été traités de manière séparée. Leur importance relative a d'abord été quantifiée. Pour cela, la proportion d'objets qui ont subi un grand déplacement a été déterminée pour chaque cellule et par année, en considérant comme appartenant à cette classe toute distance parcourue présentant une probabilité inférieure à $1 \%$ d'être due à la solifluxion. Cette proportion atteint $4 \%$ du total des déplacements enregistrés. Un test de Student indique que cette proportion ne diffère pas sensiblement sur la durée de l'expérience selon que les objets soient des silex ou des ossements. Ce n'est pas le cas pour la distance maximale parcourue, qui atteint $3 \mathrm{~m}$ dans le cas des ossements (fig. 6B) alors qu'elle ne dépasse pas $1 \mathrm{~m}$ pour les silex (fig. 10A). Pour ces derniers, la distribution des déplacements suit une loi log-normale. L'analyse montre également que les grands déplacements sont d'autant plus orientés vers la pente que la distance parcourue est forte. La distribution des directions de déplacement a été ajustée à une loi normale circulaire de type Von Mises (Baschelet 1981) et l'écart-type angulaire a été déterminé. Cet ajustement a été réalisé pour trois sous-populations de manière à prendre en compte la corrélation existante entre orientation du déplacement et distance parcourue. 
15 A partir de ces données, une simulation informatique a été réalisée. Dans le programme de simulation, des déplacements sont calculés de façon à obtenir une distribution des distances parcourues et des directions de mouvement qui présentent les mêmes caractéristiques statistiques que celles des données expérimentales. Le programme procède par boucle. A chaque itération, qui représente un an de fonctionnement de l'expérience TRANSIT, des valeurs de déplacement de mêmes caractéristiques que les données expérimentales sont générées. Ainsi, pour chaque année simulée, les coordonnées $\mathrm{X}, \mathrm{Y}$ des objets deviennent (l'axe des $\mathrm{Y}$ étant parallèle à la pente) :

$X_{n+1}=X_{n}+d \cos a$

$\mathrm{Y}_{\mathrm{n}+1}=\mathrm{Y}_{\mathrm{n}}+\mathrm{d} \sin \mathrm{a}$

où $\mathrm{d}$ : distance de déplacement $(\mathrm{en} \mathrm{cm}$ )

$\mathrm{a}$ : valeur de l'angle entre la direction de déplacement et la pente (en radians)

La programmation a été réalisée avec le logiciel Data Desk version 6.1, qui offre une interface graphique permettant de suivre l'évolution de la répartition des objets à chaque étape. Plusieurs hypothèses ont été introduites dans cette programmation. La principale concerne la distribution des vestiges avant qu'ils ne soient déplacés. La configuration initiale retenue ici est celle d'un amas de taille; elle est motivée par les raisons suivantes :

- Sur les sites de Petit-Bost et Croix-de-Canard, les données archéologiques ont mis en évidence des activités de débitage du silex. La présence d'amas ultérieurement rendus illisibles par les perturbations naturelles est donc une hypothèse plausible.

- Cette configuration a été utilisée pour d'autres expériences (Schick 1986 ; Barton et Bergman 1982). Les résultats des différentes expériences peuvent donc être comparés.

- Ce type de structure est bien connu et a été reproduit expérimentalement (Newcomer et Sieveking 1980 ; Hansen et Madsen 1983 ; Boëda et Pellegrin 1985).

\section{Analyse Dimensionnelle de la Variance}

De manière à pouvoir quantifier les modifications de la répartition des objets tout au long de la simulation, nous avons fait appel à " l'Analyse Dimensionnelle de la Variance " proposée par Whallon (1973). Cette méthode est utilisée en archéologie pour analyser la répartition spatiale d'objets dans un site et identifier l'existence et la taille des concentrations. Le plan de répartition des objets est découpé en surfaces élémentaires (ici $1 / 64 \mathrm{e}$ de $\mathrm{m} 2$, soit $12,5 \mathrm{~cm} \times 12,5 \mathrm{~cm}$ ). Les surfaces élémentaires sont ensuite doublées, puis quadruplées, etc... et la variance de la distribution des vestiges est calculée à chaque étape (la méthode de calcul est exposée dans Whallon 1973). Le nombre moyen d'objets par unité de surface est également calculé, ce qui permet d'établir un rapport variance / moyenne. La valeur de ce rapport est élevée si des concentrations d'objets existent ; elle est en revanche faible dans le cas d'une répartition uniforme du matériel archéologique, c'est-à-dire si chaque surface élémentaire d'une taille donnée contient le même nombre d'objets (fig. 11). Le rapport est proche de 1 lorsque la répartition est «aléatoire», c'est-à-dire lorsque la distribution du contenu des surfaces élémentaires suit une loi de Poisson (Djindjian 1991). 
Figure 11 - Diagramme de Whallon (1973). La courbe en gras correspond à l'amas expérimental utilisé dans la simulation.

Figure 11 - Whallon's (1973) diagram. The bold curve corresponds to the knapping spot used in the simulation.

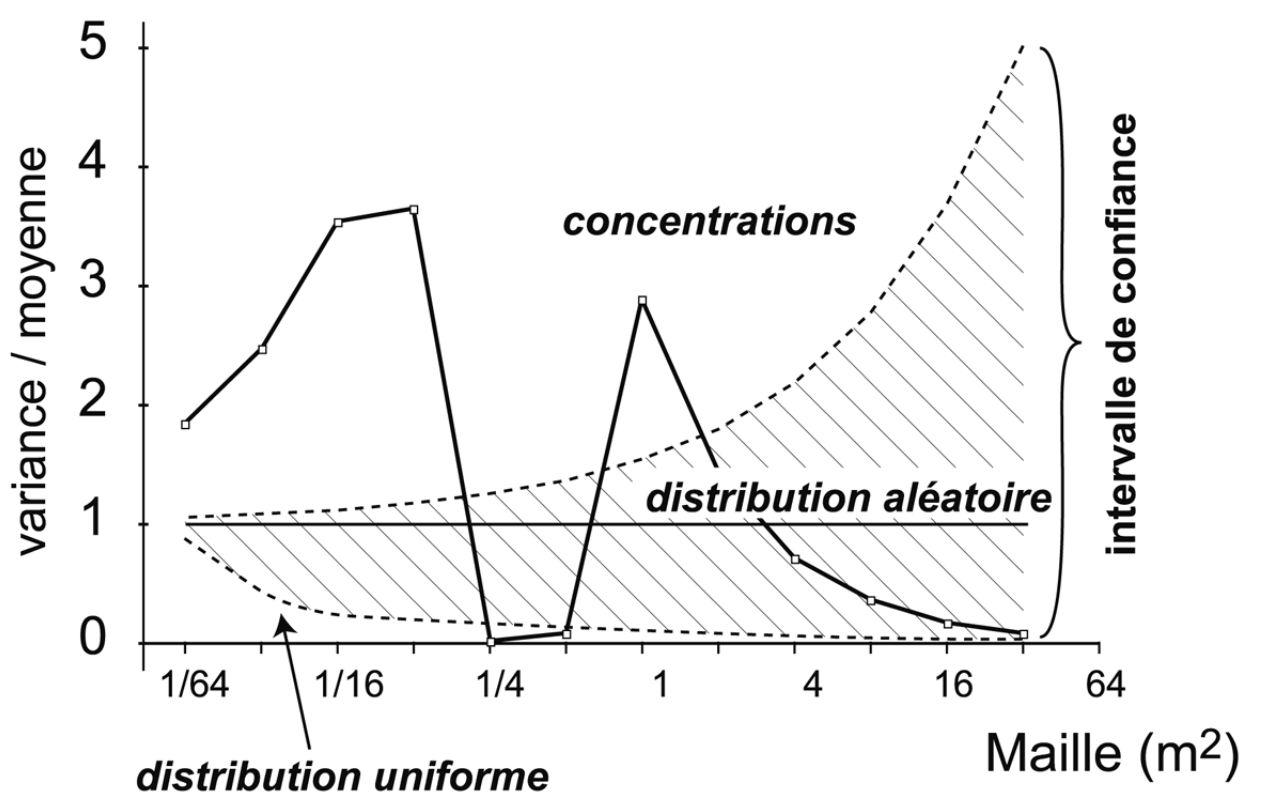

Un graphique rapport variance / moyenne en fonction de la taille de la surface élémentaire (que l'on appellera graphique de Whallon) permet d'identifier les mailles qui font le mieux ressortir les concentrations de vestiges ou les zones vides. Les premières correspondent aux pics sur le graphique. Le caractère significatif d'une concentration est testé à l'aide du Chi-carré. On peut reporter ainsi sur le graphique une zone de valeurs pour lesquelles les concentrations ne sont pas statistiquement significatives. La figure 11 présente le graphique obtenu pour l'amas de débitage utilisé dans la simulation. Deux pics ressortent sur ce graphique : le premier correspond à une maille de $1 / 16 \mathrm{~m}^{2}$ (soit $0,25 \times 0,25 \mathrm{~m}$ ) et reflète la présence de sous-concentrations au sein de l'amas, tandis que le second apparait pour une maille de $1 \mathrm{~m} 2$ et correspond à l'amas lui-même. Le rapport variance / moyenne est indépendant de la surface totale de l'aire étudiée. Il dépend en revanche de la position des concentrations par rapport à la grille de décompte utilisée (Djindjian 1991). Ceci explique sur la figure 11 la faible valeur obtenue pour la maille de $1 / 4 \mathrm{~m}^{2}$ : la concentration, qui est placée au centre d'un carré de $1 \mathrm{~m}$ de côté, se retrouve répartie de façon égale entre les quatre sous-carrés de $50 \mathrm{~cm}$ de côté.

\section{Résultats de la simulation}

Les modifications simulées d'un amas composé de 120 éléments font apparaître deux composantes (fig. 12). La première consiste en une translation vers l'aval du centre de l'amas, qui atteint environ $4 \mathrm{~m}$ par siècle. Les mouvements «accidentels», bien que peu nombreux (4\%), contribuent pour une large part (25\%) au déplacement général (fig. 13). La seconde composante est une déformation, qui se traduit par un accroissement de la taille de l'amas. La dispersion des objets, plus importante vers l'aval que dans la direction perpendiculaire, donne naissance à une concentration allongée dans la pente 
en forme d'ellipse ou d'éventail, avec une partie amont plus dense que la partie aval. Cette dispersion provoque un affaiblissement progressif de la concentration, bien visible sur les graphiques de Whallon (fig. 14). Cinquante à cent années simulées suffisent pour que la distribution des objets ne puisse plus être distinguée d'une répartition aléatoire, tandis que les sous-concentrations initiales ne sont plus perceptibles au-delà de vingt années. Ce phénomène de diffusion et d'homogénéisation peut également être illustré par l'évolution du nombre d'objets par unité de surface au cours de la simulation (fig. 15).

Figure 12 - Évolution d'un amas de débitage A composé de 120 pièces après 20 (B) et 50 (C) années de solifluxion simulée.

Figure 12 - Evolution of a knapping spot A (120 artefacts) after 20 (B) and 50 (C) years of simulated solifluction.
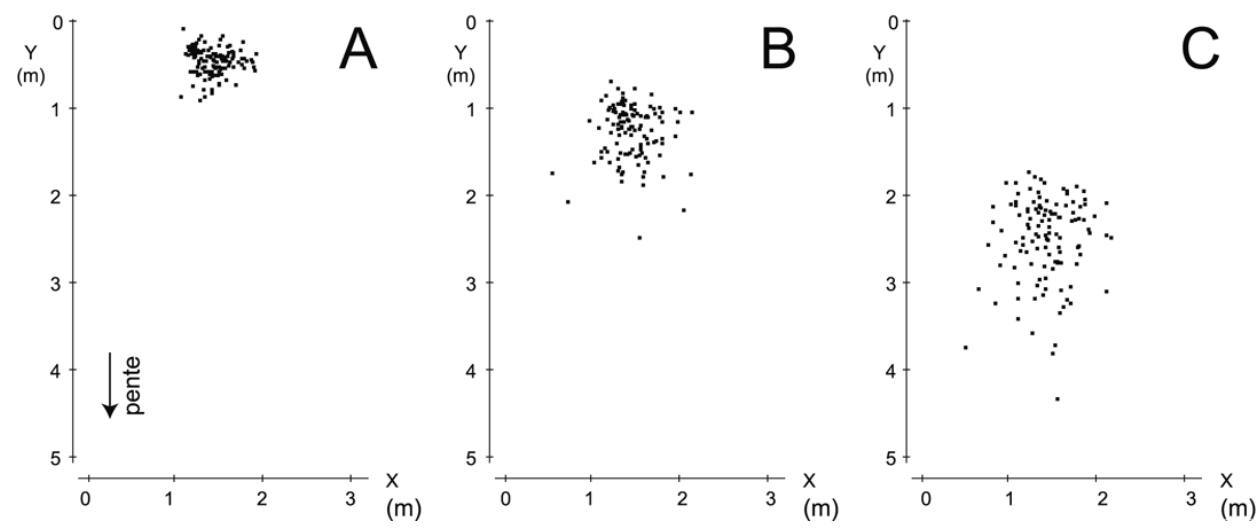

Figure 13 - Comparaison entre le déplacement simulé provoqué par la seule solifluxion et la solifluxion additionnée des mouvements accidentels.

Figure 13 - Comparison between the simulated displacement due to solifluction only and to solifluction plus the accidental mouvements.

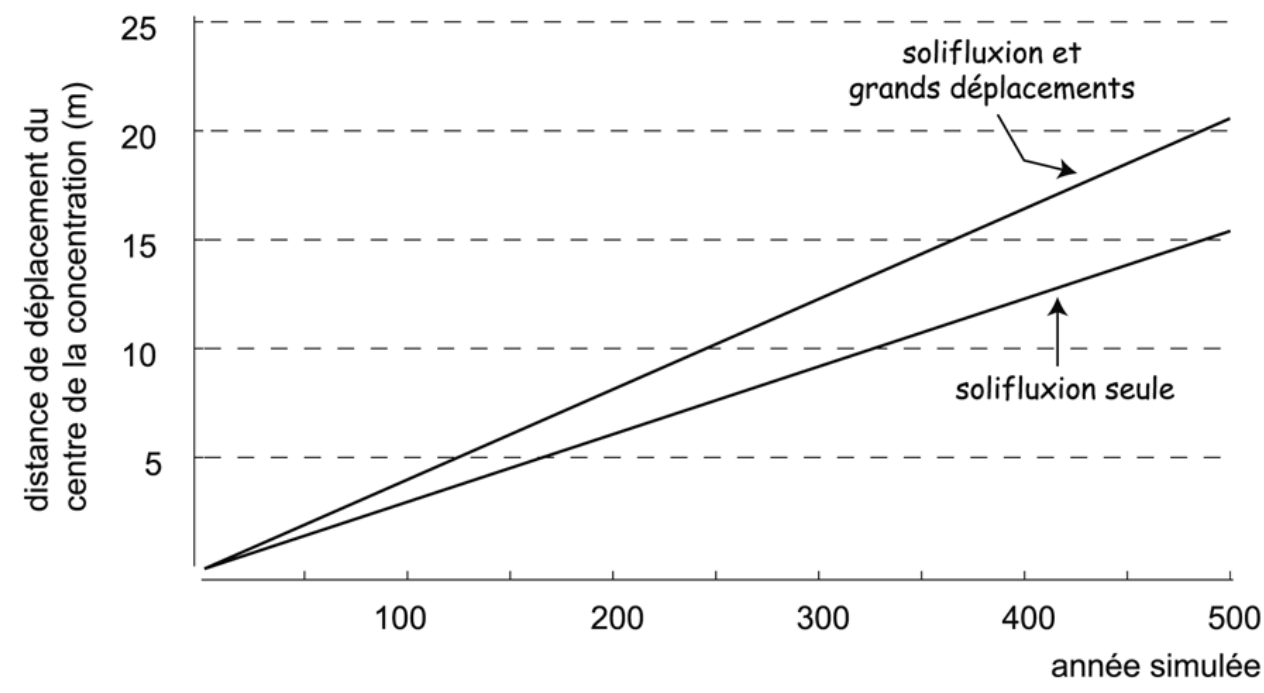


Figure 14 - Évolution de l'amas et du diagramme de Whallon correspondant au cours de la simulation. L'échelle des plans de répartition des objets est graduée en mètres.

Figure 14 - Evolution of the knapping spot and the Whallon's diagram during the simulation. The scale of the artefact maps is in metres.
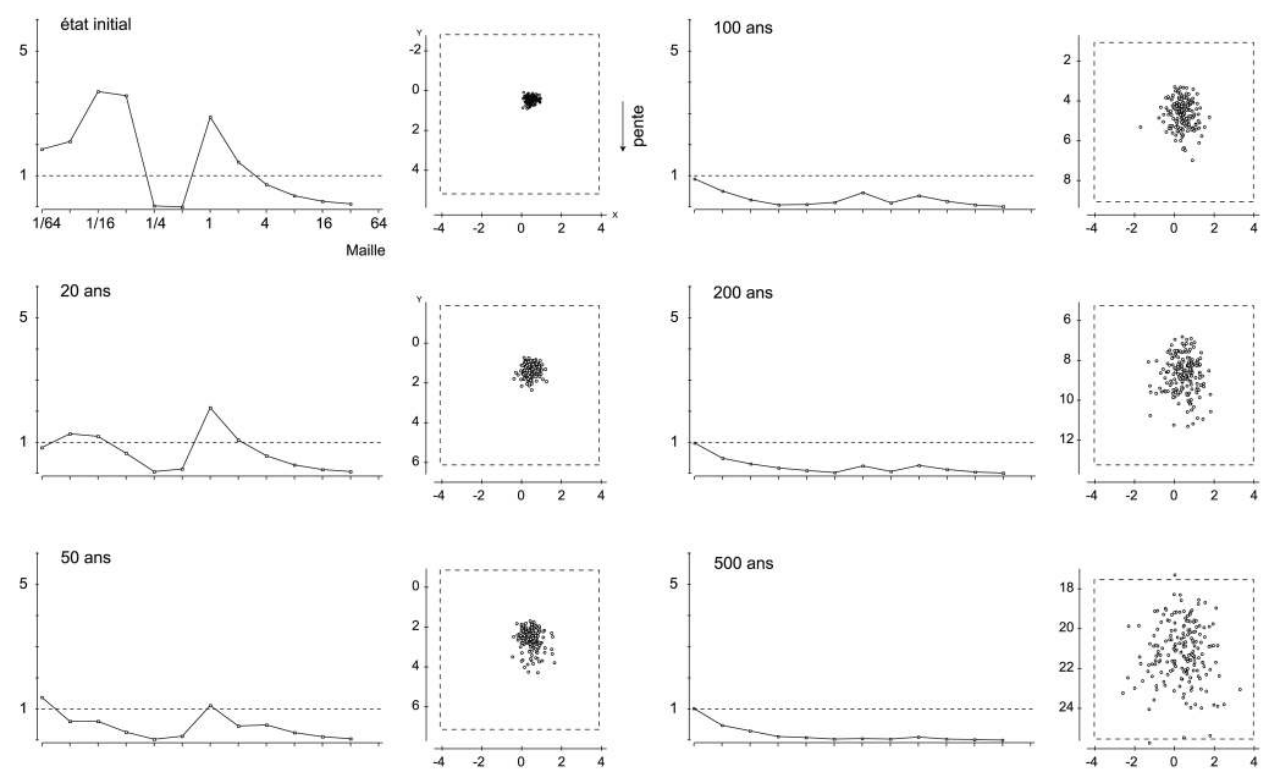

Figure 15 - Évolution de la concentration maximale d'objets par $\mathrm{m}^{2}$ au cours de la simulation pour des surfaces de $0,25,1$ et $4 \mathrm{~m}^{2}$ centrées sur le cœur de l'amas. Chaque courbe représente la moyenne de trente essais ; l'écart-type est indiqué en grisé.

Figure 15 - Evolution of the maximum artefact concentration $\left(\mathrm{N} / \mathrm{m}^{2}\right)$ during the simulation for $0.25,1$ and $4 \mathrm{~m}^{2}$ surfaces centered on the knapping spot. Each curve is the mean of thirty runs. The mean square is in grey.

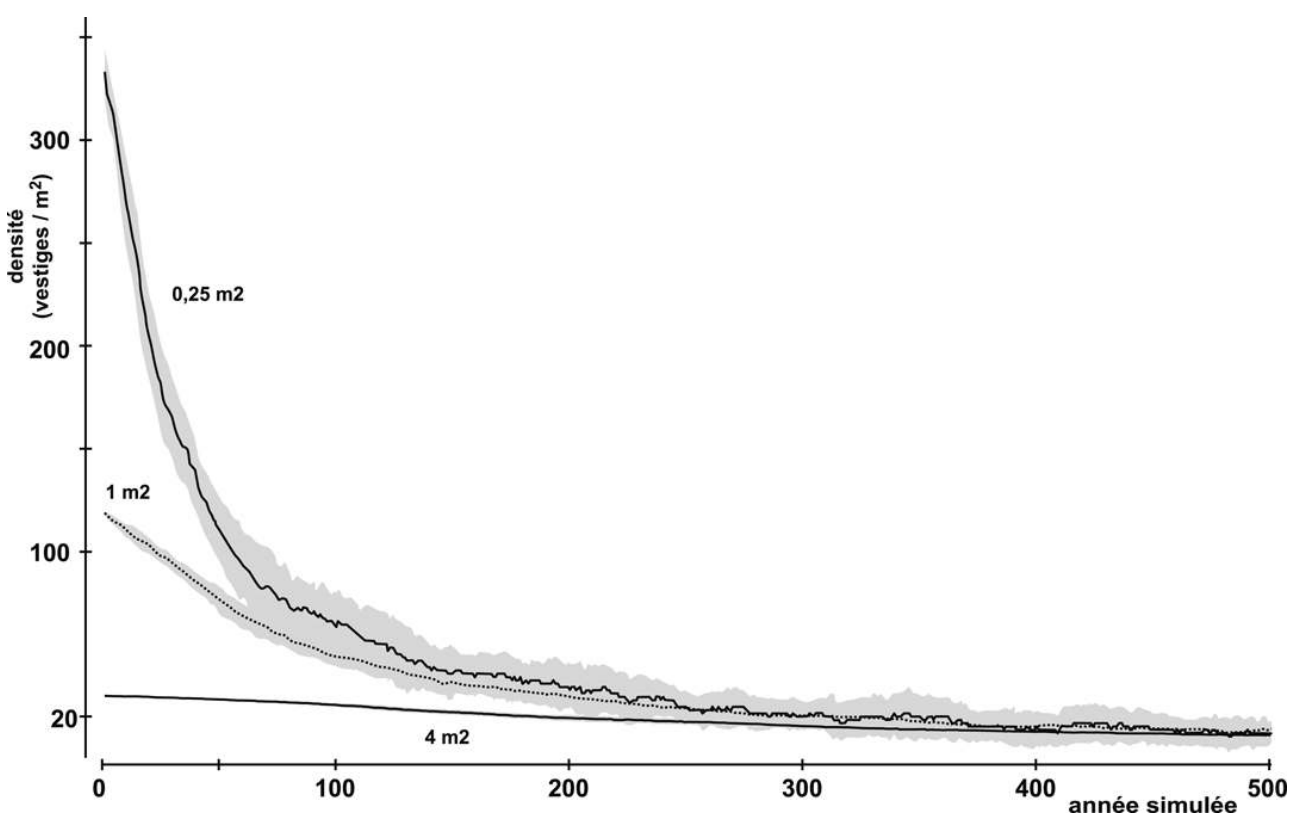




\section{Confrontation avec les données archéologiques}

\section{Petit-Bost}

La comparaison entre la concentration archéologique et la simulation montre de grandes similitudes dans la distribution spatiale des vestiges pour des déplacements correspondant à quelques centaines d'années. Ces similitudes concernent :

- La forme générale de la concentration. Celle-ci est étirée et son axe d'allongement est conforme à la pente.

- La distribution des objets au sein de la concentration. La moitié amont est beaucoup plus dense que la moitié aval. Cette dernière présente des limites floues, le nombre d'objets par unité de surface diminuant progressivement vers l'aval.

21 L'hypothèse d'un amas de débitage déformé par la solifluxion rend donc bien compte des caractéristiques générales de la concentration d'objets découverte sur le site de Petit-Bost, autant pour la répartition spatiale que pour la présence d'une orientation préférentielle des vestiges dans la pente. La simulation ne permet cependant pas d'estimer précisément le degré de déformation de l'amas initial. En effet, l'asymétrie de la concentration peut être comparée avec celle obtenue dans la simulation pour 20 à 100 ans tandis que la dispersion maximale des vestiges dans la pente, qui atteint une dizaine de mètres, suggère un déplacement de plus grande ampleur, obtenu après environ 500 ans. Le graphique de Whallon ne met pas en évidence de concentration remarquable (fig. 16). On note cependant que le rapport variance / moyenne augmente légèrement pour les mailles de 1 et $2 \mathrm{~m}$ et que, sur cette base, la répartition des objets peut être rapprochée de celle obtenue par simulation pour 200 ans.

Figure 16 - Diagramme de Whallon pour le niveau 1 de Petit-Bost. Figure 16 - Whallon's diagram for level 1, Petit-Bost.

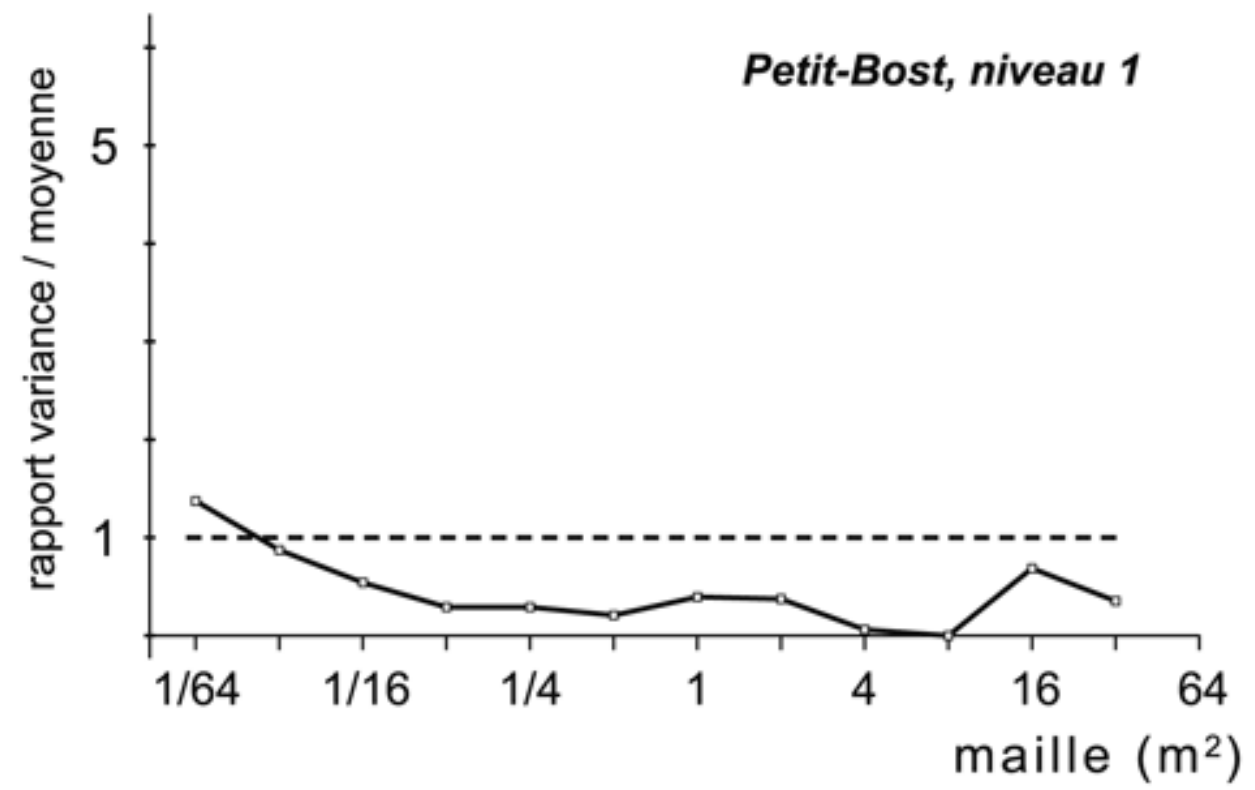

Afin de préciser les caractéristiques de la répartition des objets, nous avons analysé séparément les remontages constitués d'un nombre significatif de pièces. Trois d'entre eux, composés respectivement de 118, 73 et 31 éclats, ont été pris en compte. La 
distribution des objets qui composent ces trois ensembles est globalement conforme à celle de la concentration principale : elle montre une dispersion des pièces essentiellement dans la pente, dont l'ampleur est comparable pour les trois remontages. Pour le remontage 1 , la plus forte densité de vestiges est localisée à l'amont (fig. 17). Les graphiques de Whallon pour les remontages 2 et 3 sont comparables à ceux obtenus dans la simulation pour 200 ans, comme dans le cas de la concentration principale. En revanche, dans le cas du premier remontage (118 pièces), le graphique se rapproche de celui obtenu pour une vingtaine d'années. A partir de l'évolution du nombre d'objets par unité de surface (fig. 15), sont établies les durées nécessaires pour obtenir une densité de vestiges comparable à celle mesurée au centre de chaque ensemble remonté (fig. 18). Plusieurs informations se dégagent de cette figure :

- Les valeurs obtenues pour les remontages 2 et 3 se recouvrent et suggèrent un déplacement comparable à celui produit par 100 à 250 ans de solifluxion simulée.

- La répartition des vestiges du premier remontage n'est en revanche pas compatible avec une déformation par solifluxion telle que nous l'avons simulée. Cela tient au fait qu'une forte concentration résiduelle subsiste dans la partie amont de l'ensemble, en contradiction avec l'ampleur de la dispersion des pièces à l'extérieur de cette concentration.

- L'opposition entre un «amas résiduel» dense et une dispersion importante des pièces en dehors de cet amas est perceptible pour les trois groupes de pièces remontées. Dans chaque cas, la densité pour la plus petite maille correspond à un nombre d'années plus faible que pour les mailles plus grandes. On retrouve donc, à l'échelle de chaque remontage, une certaine contradiction entre la partie amont, où est préservée une densité élevée de vestiges, et la dispersion importante vers l'aval des objets.

Figure 17 - Diagramme de Whallon pour trois remontages de Petit-Bost. Figure 17 - Whallon's diagram for three reffitings, Petit-Bost.
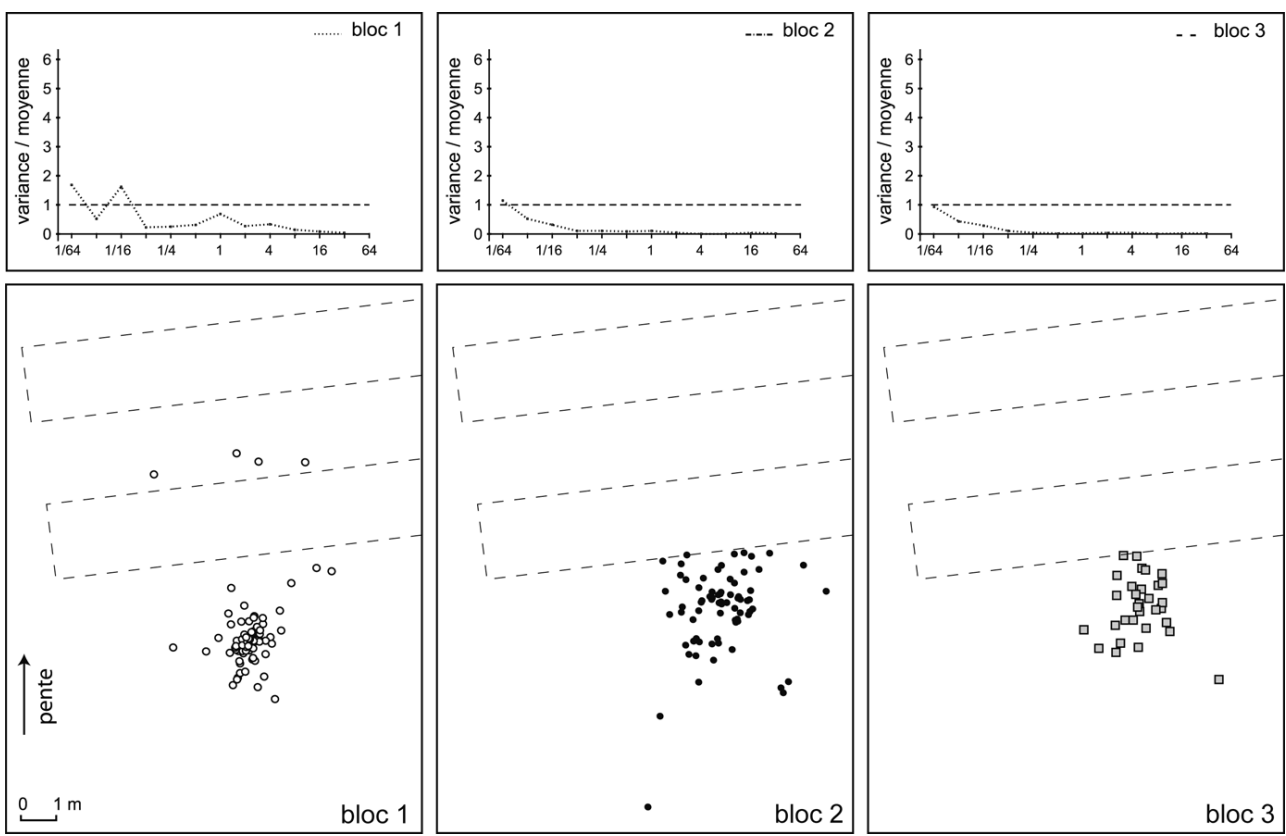
Figure 18 - Nombre d'années de solifluxion simulée nécessaire à l'obtention d'une concentration d'objets par mètre carré comparable à celles des remontages de Petit-Bost.

Figure 18 - Number of years of solifluction needed to obtain by simulation artefact concentrations / m2 similar to those of the reffitings at Petit-Bost.

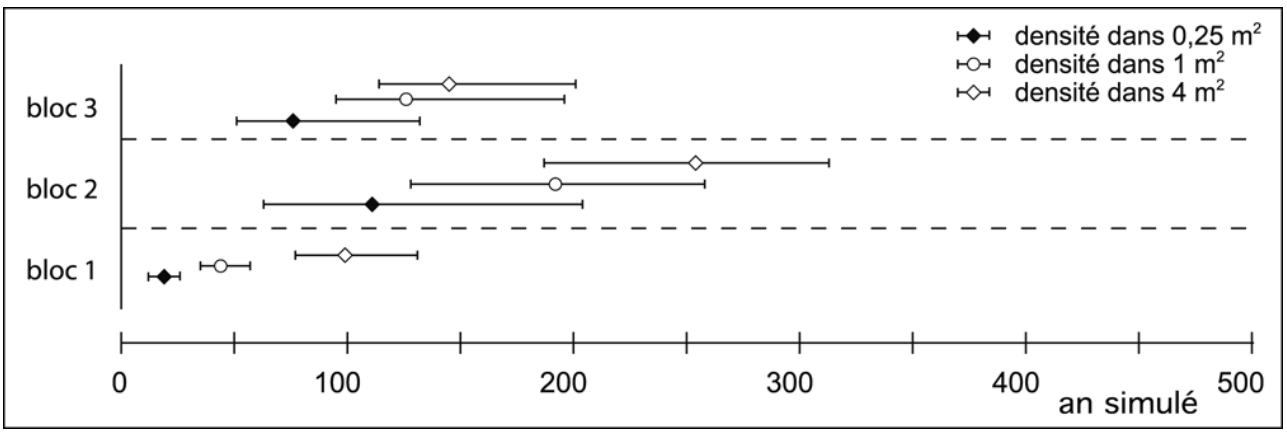

Ces observations conduisent à s'interroger sur l'influence de la disposition initiale des objets affectés par la solifluxion. En effet, plusieurs études sur la dynamique de solifluxion ont montré que la vitesse de déplacement était très dépendante de la texture du sol. Sur un même site, les mouvements mesurés dans les zones caillouteuses sont en effet toujours plus lents que ceux trouvés dans les zones à texture fine, où de plus grandes quantités de glace peuvent se former. Dans le massif de la Mortice, Coutard et Ozouf (1996) enregistrent ainsi une vitesse 2 à 10 fois supérieure dans les bandes fines que dans les bandes caillouteuses des sols striés. Un tel phénomène pourrait donc jouer un rôle significatif dans le cas d'un amas de débitage. Il pourrait notamment être à l'origine de déplacements plus importants dans la zone périphérique que dans la partie centrale de l'amas où la densité d'objets s'accroît.

\section{Croix-de-Canard}

L'analyse a porté sur le locus 2 du secteur 2, qui est le plus étendu et a livré le plus grand nombre d'objets. L'Analyse Dimensionnelle de la Variance montre qu'aucune concentration ne peut être détectée (fig. 19). A l'opposé, la répartition des vestiges tend vers une distribution uniforme pour des surfaces élémentaires de plus de $1 / 4 \mathrm{~m} 2$. Le graphique de Whallon est comparable à celui obtenu dans la simulation pour de longues durées de fonctionnement (plus de 500 ans). Contrairement à ce que l'on observe à Petit-Bost, les remontages réalisés sont très dispersés et ne forment pas de concentration claire, bien que le taux de remontage soit important (37\% du total des pièces). Les séries de pièces raccordées sont nombreuses, mais elles ne mettent chacune en jeu qu'un petit nombre d'objets (la plus importante est composée de 17 pièces). L'ensemble de ces observations suggère donc une forte dispersion et une homogénéisation complète du niveau d'occupation, qui, à la lumière de la simulation, peuvent être expliquées par l'action de la solifluxion sur une longue durée. Plusieurs raisons peuvent être invoquées pour expliquer le fait que l'intégrité de l'assemblage lithique ait été en partie préservée, malgré l'importance des transformations :

- la solifluxion ne s'accompagne pas d'un tri important des objets sur la pente et la composition de l'assemblage lithique n'a donc pas été perturbée ;

- la fouille a concerné une superficie importante ( $250 \mathrm{~m} 2$ pour le locus 2 ) ; la plus grande partie de l'assemblage initial a donc été récolté malgré sa dispersion par les processus naturels. 
Figure 19 - Diagramme de Whallon pour le secteur 2 de Croix-de-Canard. Figure 19 - Whallon's diagram, sector 2, Croix-de-Canard.

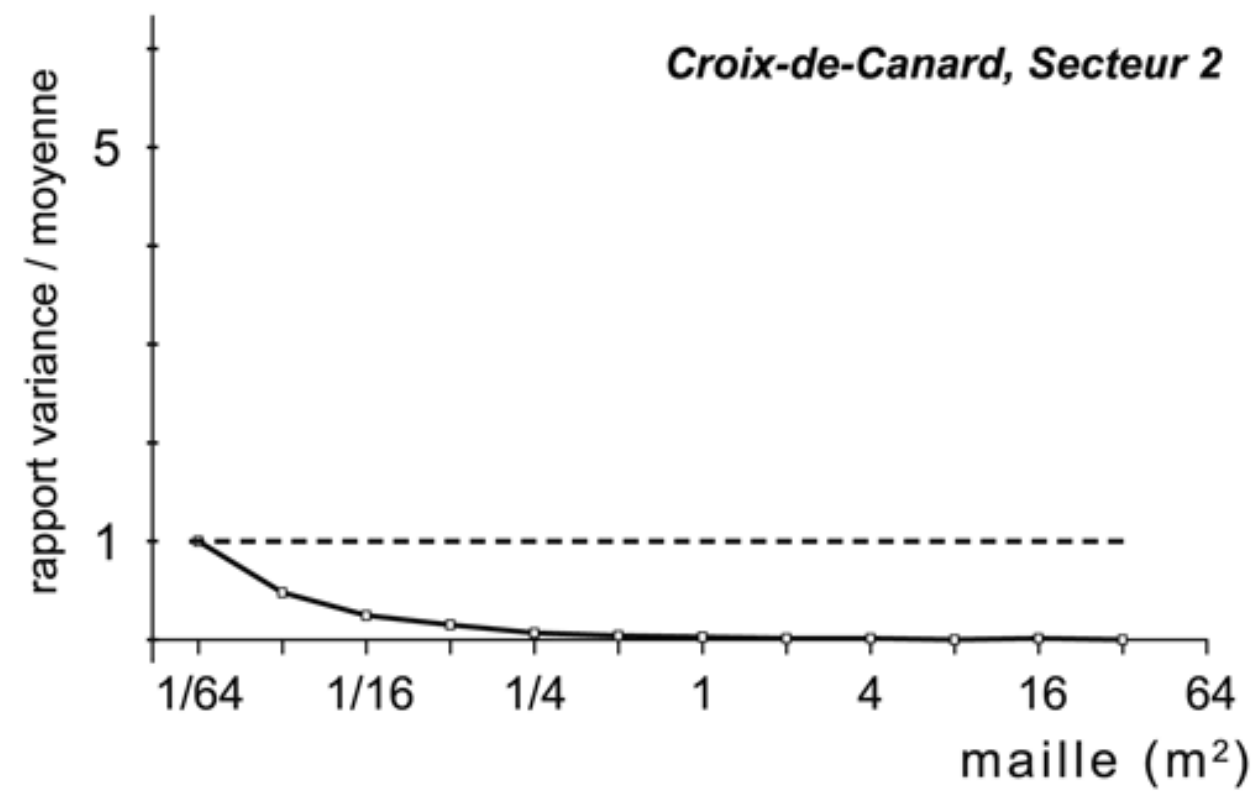

\section{Discussion}

A l'issue de la simulation, les principaux résultats obtenus montrent que, pour une concentration d'objets tel qu'un amas de débitage :

- Les premiers stades de la déformation par solifluxion se traduisent à la fois par une translation vers l'aval du centre de gravité de la concentration et par une diffusion anisotrope des objets. Cette diffusion provoque un étalement et une diminution de la densité d'objets ; l'amas prend alors une forme d'éventail allongé dans la pente. Une forte densité relique d'objets subsiste cependant dans la partie amont tandis que la partie aval a des contours très diffus. Dans le cadre des données de l'expérience TRANSIT, ces stades correspondent à 100 à 200 ans de fonctionnement ; la translation correspondante vers l'aval atteint respectivement 4 et $8 \mathrm{~m}$.

- Dans les stades ultérieurs de la déformation, la répartition du matériel tend alors à s'homogénéiser sur une grande surface et s'apparente à une distribution aléatoire des objets. Pour l'expérience TRANSIT, ceci se produit au-delà de 400 ans de fonctionnement.

La comparaison des résultats de la simulation avec la répartition des vestiges sur les sites paléolithiques de Petit-Bost et Croix-de-Canard permet de montrer que l'hypothèse d'une redistribution des objets par la solifluxion rend bien compte des observations. Cette hypothèse permet en particulier d'expliquer la coexistence dans le site de Petit-Bost de traits d'origine manifestement anthropique (la présence d'une concentration d'objets avec un fort taux de remontage, identifiée comme une aire de débitage) et de traits manifestement «naturels» (une orientation préférentielle des objets dans la pente), qui apparaissaient au premier abord inconciliables. Selon ce schéma, l'aire de débitage correspondrait à un amas déformé sur la pente. Une configuration identique peut également être envisagée pour le site de Croix-de-Canard. Il faut également noter, en ce qui concerne ce site qui reflèterait un stade «ultime» de 
dégradation, la persistance de remontages «résiduels» mettant en jeu un nombre de pièces limitées (17 pour le remontage le plus important).

Dans tous les cas, le recours à la simulation permet de comparer les différentes déformations observées sur des bases quantitatives. Il ne saurait cependant autoriser une estimation précise des durées pendant lesquelles les sites archéologiques ont été soumis à la solifluxion. Plusieurs points, en effet, limitent la portée du modèle et méritent d'être soulignés. Ils concernent un certain nombre d'hypothèses sur lesquelles repose la simulation :

- Les mesures de déplacement servant de base à la simulation ont été faites dans un milieu précis, celui de la Mortice (Alpes françaises méridionales). Le rôle relatif et l'efficacité des différents mécanismes impliqués dans la solifluxion (cryoreptation, gélifluxion, pipkrakes) sont largement tributaires des caractéristiques physiques et climatiques du milieu ; les mesures effectuées ne sont donc pas nécessairement représentatives de tous les types d'environnements où la solifluxion intervient. Par exemple, les mesures réalisées au cours de l'expérience TRANSIT ont été faites sur une pente de 10 à $14^{\circ}$ et sont appliqués à des niveaux archéologiques présentant des pentes sensiblement plus faibles. Il est possible que cet écart influe sur les vitesses de déplacement, bien que cette influence soit difficile à saisir. En effet, les niveaux archéologiques sont inclus dans des sols fins mal drainés. Dans ce type de sol, l'amélioration du drainage qui accompagne l'augmentation de la pente réduit les possibilités de formation de glace dans le sol et, en conséquence, le gonflement cryogénique lié au gel saisonnier profond. La corrélation entre la pente et la vitesse de déplacement est alors faible voire négative (Smith 1992). Le rôle de cet écart de pente ne peut d'ailleurs pas être isolé de celui des autres paramètres (la texture des sols, l'importance des précipitations et leur distribution dans l'année, la profondeur du gel, la position topographique du site, etc.), dont le jeu des interactions détermine la vitesse de déplacement (French 1976). Le modèle déduit de l'expérience TRANSIT ne doit être considéré que comme un cas particulier des déformations occasionnées par la solifluxion et il ne peut notamment servir à une estimation précise de la durée pendant laquelle un site a été soumis à ce processus. Par ailleurs, dans les milieux naturels, d'autres processus de versant comme le ruissellement ou les avalanches sont souvent associés à la solifluxion et contribuent à des degrés divers aux mouvements des objets à la surface du sol. L'influence de ces processus est souvent due à des événements de fréquence faible (des orages ou des chutes de neige exceptionnels, de temps de retour centennal) mais dont l'impact morphogénétique est important. La faible durée de l'expérience TRANSIT (cinq ans) ne permet pas de prendre en compte ce type de phénomènes, dont les traces dans le sédiment peuvent être très fugaces car effacées par les cycles de gel-dégel ultérieurs.

- La simulation réalisée a nécessité certaines simplifications du fonctionnement observé en milieu naturel. Ainsi, la variabilité latérale des vitesses de déplacement au sein des coulées, caractérisées par une progression plus lente au niveau du front et des bords que dans la partie centrale, a été ignorée. La prise en compte de cette variabilité aurait conduit à mettre en évidence des déformations plus complexes. Ce phénomène est probablement sensible dès que l'on s'adresse à des surfaces de plusieurs mètres-carrés. Par ailleurs, l'enfouissement de la fraction grossière se produit surtout au niveau des fronts et les différents secteurs d'un niveau archéologique implanté sur une coulée risquent de ne pas être affectés de la même manière par la déformation.

- Les données de l'expérience TRANSIT ne permettent pas d'aborder l'influence de la disposition initiale des objets sur la vitesse de déplacement, en particulier lorsqu'ils sont disposés en amas. Or, la relation entre vitesse et granulométrie du sol est un fait bien connu. 
Comme cela a été discuté plus haut, nous pensons que cette influence peut expliquer la distorsion entre la simulation et la distribution des vestiges observée à Petit-Bost.

\section{Conclusions} transformations provoquées par la solifluxion sur des assemblages archéologiques. Elle permet notamment d'obtenir une idée précise des configurations spatiales du matériel archéologique qui sont susceptibles d'apparaître pour des temps variés d'exposition d'un site à ce processus.

Malgré un certain nombre de limitations, l'application aux sites du Paléolithique moyen de Petit-Bost et de Croix-de-Canard (Dordogne, France) montre que la simulation rend globalement bien compte de cas archéologiques et permet d'expliquer de manière simple un ensemble d'observations difficilement compréhensibles de manière purement intuitive. Elle suggère également que certains critères, comme la présence de concentrations évidentes d'objets ou un pourcentage élevé de pièces remontant entre elles, ne sont pas nécessairement pertinents pour déterminer le caractère " non déplacé " d'un assemblage archéologique. Cela est le cas pour le niveau 1 de Petit-Bost, où des concentrations remarquables s'observent alors que l'organisation spatiale des vestiges a été partiellement dégradée au cours de l'enfouissement. Cela est également le cas pour le secteur 2 de Croix-de-Canard, où un taux de remontage important est observé alors que la disposition des vestiges est significativement influencée par la solifluxion.

La simulation a également le mérite de mettre en évidence un certain nombre de points qui nécessiteraient de plus amples investigations de manière à mieux appréhender les processus d'enfouissement des sites en contexte de solifluxion, comme le rôle de la disposition initiale des objets ou l'influence des fronts de coulées.

\section{BIBLIOGRAPHIE}

ARAUJO A. G. M. et MARCELINO J. C., 2003 - The role of armadillos in the movement of archaeological materials : an experimental approach. Geoarchaeology, 18 : 433-460.

ASCHER R., 1968 - Time's arrow and the archaeology of a contemporary community. Settlement archaeology, K. C. Chang (Ed.), National Press Books, Palo Alto : 43-52.

BARTON R. N. E. et BERGMAN C. A., 1982 - Hunters at Hengistbury : some evidence from experimental archaeology. World archaeology, $14: 237-248$.

BASCHELET E., 1981 - Circular statistics in biology. Academic Press, Londres, 374 p.

BENN D. I., 1994 - Fabric shape and the interpretation of sedimentary fabric data. Journal of Sedimentary Research, A64, 4 : 910-915.

PALEO, 15 | 2003 
BERTRAN P., 1994 - Dégradation des niveaux d'occupation paléolithiques en contexte périglaciaire et implications archéologiques. Paléo, 6 : 285-302.

BERTRAN P., FRANCOU B. et PECH P., 1993 - Stratogenèse assistée à la dynamique des coulées à front pierreux en milieu alpin, La Mortice, Alpes méridoniales, France. Géographie physique et Quaternaire, 47 : 93-100.

BERTRAN P., FRANCOU B. et TEXIER J. P., 1995 - Stratified Slope Deposits : the Stone-banked Sheets and Lobes Model. Steepland Geomorphology, O. Slaymaker (Ed.), Wiley \& Sons, Londres : 147-169.

BERTRAN P. et LENOBLE A., 2002 - Fabriques des niveaux archéologiques : méthode et premier bilan des apports à l'étude taphonomique des sites paléolithiques. Paléo, 14 : 13-28.

BINFORD L., 1981 - Behavorial archaeology and the «Pompei premise». Journal of Anthropological Research, $37: 195-208$.

BOËDA E. et PELLEGRIN J., 1985 - Approche expérimentale des amas de Marsangy. Les amas lithiques de la zone N19 du gisement magdalénien de Marsangy : approche méthodologique par l'expérimentation. Archéologie expérimentale, cahier 1, Association pour la promotion de l'archéologie de Bourgogne, Archéodrome : 19-36.

BOWERS P. M., BONNICHSEN R. et HOCH D. M., 1983 - Flake dispersal experiments : non-cultural transformation of the archaeological record. Antiquity, $48: 553-572$.

BUTZER K., 1982 - Archaeology as human ecology. Cambridge University Press, Cambridge, 364 p.

CAHEN D. et MOEYERSONS J., 1977 - Subsurface movements of stone artefacts and their implications for the prehistory of Central Africa. Nature, $266: 812-815$.

CAINE N., 1968 - The log-norma distribution and rates of soil movement : an example. Revue de géomorphologie dynamique, $18: 4-46$.

COUCHOUD I., 2002 - Processus géologiques de formation du site moustérien du Roc de Marsal. Mémoire de DEA en Anthropologie, Université Bordeaux 1, 67 p.

COUTARD J. P. et OZOUF J. C., 1996 - Modalités de la cryoreptation dans les Massifs du Chambeyron et de la Mortice, Haute-Ubaye, Alpes françaises du Sud. Permafrost and periglacial processes, $7: 21-51$.

DJINDJIAN F., 1991 - Méthode pour l'archéologie. A. Colin, Paris, 401 p.

FRENCH H. M., 1976 - The Periglacial environment. Longman, Harlow, 309 p.

HANSEN P. V. et MADSEN B., 1983 - Flint axe manufacture in the Neolithic. An experimental investigation of a flint axe manufacture site at Hasprup Vaenget, East Zealand. Journal of Danish Arcaheology, 2 : 43-59.

JOHNSON D. L., 2002 - Darwin would be proud : bioturbation, dynamic denudation, and the power of theory in science. Geoarchaeology, 17 : 7-40.

NEWCOMER M. H. et SIEVEKING G. de G., 1980 - Experimental flake scatter-patterns : a new interpretative technique. Journal of Field Archaeology, 7 : 345-352.

SCHICK K., 1986 - Stone Age in the Making : Experiments in the Formation and Transformation of Archaeological Occurences. Bar international series n 319, Oxford, 313 p.

SCHIFFER M. B., 1972 - Archaeological context and systemic context. American Antiquity, 37 : 156-165. 
SCHIFFER M. B., 1983 - Toward the identification of site formation processes. American Antiquity, $48: 675-706$.

SCHIFFER M. B., 1987 - Formation processes of the archaeological record. University of New Mexico Press, Albuberque, $427 \mathrm{p}$.

SMITH D. J., 1992 - Long-term rates of contemporary solufluction in the Canadian Rocky Moutains. Periglacial Geomorphology, Proceedings of the 22nd Annual Binghampton Symposium in Geomorphology, J. C. Dixon et A. D. Abrahams (Eds.), Wiley : 230-221.

STEINBERG J. M., 1996 - Ploughzone sampling in Denmark : isolating and interpreting site signatures from disturbed context. Antiquity, 70 : 368-392.

TEXIER J. P., 2001 - Sédimentogenèse des sites préhistoriques et représentativité des datations numériques. Datation, XXIe rencontres internationales d'archéologie et d'histoire d'Antibes, J.-N. Barrandon, P. Guibert et V. Michel (Eds.), Editions APDCA, Antibes : 159-175.

TEXIER J. P., BERTRAN P., COUTARD J. C., FRANCOU B., GABERT P., GUADELLI J. L., OZOUF J. C., PLISSON H., RAYNAL J. P. et VIVENT D., 1998 - TRANSIT, an experimental archaeological program in periglacial Environment : problematic, methodology, first results. Geoarchaeology, 13 : 433-473.

TODISCO D., 1999 - Vitesses et contrôle climatique des coulées à front pierreux de la Mortice (HauteUbaye, Alpes françaises du sud). Mémoire de maîtrise en Géographie, Université de Paris-Sorbonne, $210 \mathrm{p}$.

TODISCO D., BERTRAN P. et PECH P., 2000 - Déplacements superficiels et contrôle climatique des coulées à front pierreux de la Mortice, Haute-Ubaye, Alpes françaises du Sud. Permafrost and periglacial processes, $11: 97-108$.

WATERS M. R., 1992 - Principles of geoarchaeology. The University of Arizona Press, Tucson, 399 p. WHALLON R., 1973 - Spatial analysis of occupation floors. I : application of dimensional analysis of variance. American Antiquity, 38 : 266-277.

\section{RÉSUMÉS}

L'étude taphonomique de deux sites du Paléolithique moyen, Petit-Bost et Croix-de-Canard, récemment découverts dans des colluvions près de Périgueux (Dordogne, France) à l'occasion de travaux d'archéologie préventive, a mis en lumière la difficulté d'apprécier le rôle respectif des facteurs anthropiques et naturels, en l'occurrence la solifluxion, sur la répartition spatiale des vestiges. De manière à mieux appréhender les transformations occasionnées par la solifluxion périglaciaire, c'est-à-dire par la lente reptation du sol provoquée par les alternances de gel-dégel, une simulation informatique a été réalisée à partir de mesures de déplacement enregistrées dans un milieu actif à La Mortice (Alpes françaises méridionales, $3100 \mathrm{~m}$ d'altitude) dans le cadre du programme TRANSIT. Les résultats de la simulation montrent que, pour une concentration d'objets de type amas de débitage, les premiers stades de la déformation se traduisent à la fois par une translation vers l'aval du centre de gravité de la concentration et par une diffusion anisotrope des objets. L'amas prend alors une forme d'ellipse allongée dans la pente, avec une forte densité relique d'objets dans la partie amont. Ce type de configuration est obtenu après 100 à 200 ans de fonctionnement dans les conditions du site expérimental de La Mortice. Dans les stades ultérieurs de la déformation, la répartition du matériel tend à s'homogénéiser et s'apparente à une distribution aléatoire sur une grande surface. La comparaison entre la simulation et les configurations archéologiques rencontrées sur les sites de Petit-Bost et Croixde-Canard montre une bonne adéquation. En ce qui concerne le premier, l'hypothèse d'un 
déplacement limité par la solifluxion rend notamment bien compte de la coexistence de traits anthropiques (présence de concentrations d'objets) et de traits manifestement naturels (orientation préférentielle des objets dans la pente). Pour le second site, l'hypothèse d'une action prolongée de la solifluxion ayant conduit à une homogénéisation de la répartition des vestiges peut être proposée.

The taphonomic study of Petit-Bost and Croix-de-Canard, two Middle Palaeolithic sites that have been recently discovered near Périgueux (Dordogne, France) in a colluvial context, has enlightened the difficulty to adequately appreciate the relative role of cultural and natural processes on their spatial patterning. Solifluction, i.e. a slow soil creep due to repeated freezing and thawing, is thought to have played a significant role in site formation. Since the nature of the modifications induced by solifluction is still poorly understood, a simulation has been made using the data on soil movements recorded at La Mortice (French southern Alps, $3100 \mathrm{~m}$ elevation) in a modern periglacial environment. The results show that, for a knapping spot, the first steps of deformation are typified both by a downslope translation of the spot centre and by an anisotropic diffusion of the artefacts. The knapping spot become elongated along the slope, with a dense relic concentration of artefacts in the upslope part. This kind of pattern has been obtained after 100 to 200 years of simulated displacement according to the climatic and soil conditions that characterise La Mortice site. The ultimate stages of deformation show that the artefact distribution tends to homogenise on larger surfaces and resembles that of a random distribution. These simulated patterns closely fit those observed in archaeological sites. At PetitBost, the hypothesis of limited solifluction explains accurately the association of both cultural (artefact concentrations) and natural (artefact preferred orientation) features. At Croix-deCanard, a long-lasting activity of solifluction can be proposed.

\section{INDEX}

Mots-clés : processus de formation des sites, solifluxion, simulation informatique, Paléolithique moyen, Aquitaine

Keywords : site formation processes, solifluction, simulation, Middle Palaeolithic, southwestern France

\section{AUTEURS}

\section{A. LENOBLE}

Institut de Préhistoire et de Géologie du Quaternaire, Université de Bordeaux 1, avenue des facultés, 33405 Talence cedex, France.

\section{P. BERTRAN}

Institut de Préhistoire et de Géologie du Quaternaire, Université de Bordeaux 1, avenue des facultés, 33405 Talence cedex, France.

INRAP, antenne Grand Sud-Ouest, Centre d'activité les Echoppes, 156 avenue Jean Jaurès, 33600

Pessac, France.

\section{F. LACRAMPE}

Institut de Préhistoire et de Géologie du Quaternaire, Université de Bordeaux 1, avenue des facultés, 33405 Talence cedex, France. 


\section{BOURGUIGNON}

INRAP, antenne Grand Sud-Ouest, Centre d'activité les Echoppes, 156 avenue Jean Jaurès, 33600 Pessac, France.

\section{DETRAIN}

Institut de Préhistoire et de Géologie du Quaternaire, Université de Bordeaux 1, avenue des facultés, 33405 Talence cedex, France.

INRAP, antenne Grand Sud-Ouest, Centre d'activité les Echoppes, 156 avenue Jean Jaurès, 33600 Pessac, France. 